Aus der Chirurgischen Universitätsklinik in Frankfurt a. M. (Direktor: Prof. Dr. V. Schmieden.)

\title{
Die chirurgische Topographie des wachsenden Kropfes.
}

\author{
Von Professor Dr. Heinrich Klose, Oberarzt.
}

(Mit 3 Abbildungen.)

\section{A. Einleitung.}

In der folgenden kleinen Studie habe ich den Versuch gemacht, die anatomischen Ausbreitungsmöglichkeiten des Kropfes in ein praktisch brauchbares System zu bringen. Die Untersuchungen sind nicht so entstanden, daß ich mir das Thema gewissermaßen freiwillig wählte, sondern das chirurgische und pathologisch-anatomische Interesse für dieses Gebiet, das mich seit vielen Jahren lebhaft beschäftigt, legte mir gleichsam Fragen und Antworten auf den Tisch. Das Bedürfnis nach einer Verständigung ist in der Tat groß, und dazu ist es notwendig, daß für die feststehenden Wachstumstypen, denen wiederum typische Operationsmethoden entsprechen, klare Begriffe eingeführt werden.

Ich ging von einer sicheren Voraussetzung aus, der nämlich, daß wir $z$ wei histogenetisch grundverschiedene Kropfarten kennen: die Struma diffusa und die Struma nodosa. Diese Tatsache bildet den Grundpfeiler der Kropflehre und bringt sofort Klarheit und System in die Wirrnisse der alten Anschauungen, die aus jedem Stadium der Entwicklung und Degeneration eine besondere Kropfform konstruierten. Ich lasse hier absichtlich jene Veränderungen der Schilddrüse unberücksichtigt, die als Struma maligna und inflammatoria auch mit unter dem Namen „Kropf“ zusammengefaßt werden.

Die Struma diffusa ist die Grundlage der Basedowschen Krankheit, die Struma nodosa ist die Grundlage des alläglichen gewöhnlichen Kropfes, sie basedowifiziert nur unter 
besonderen Bedingungen. Die Struma diffusa stellt eine zelluläre und funktionelle Hypertrophie oder besser hypertrophische Dystrophie der Schilddrüse dar, die sowohl einzelne Partien, wie das ganze Organ befallen kann. Die Struma nodosa ist ein Adenom der Schilddrüse, also vom Standpunkte des pathologischen Anatomen eine Gewebs miBbildung mit Geschwulstcharakter, die sich nach den verschiedenen Ländern in bezug auf die Zahl und die Größe der Knoten verschieden verhält.

Die Struma nodosa nun kann sich an die Funktionen des Mutterbodens erinnern und Kolloid absondern. Sie kann in dieser Eigenschaft einerseits cystisch entarten, verkalken oder narbig schrumpfen. Andererseits kann sie dieselben Umwandlungen im Sinne einer Dysfunktion erleiden, wie sie für das Mutterorgan beschrieben wurden, so daß wir alsdann ein Bild erhalten, das als Struma nodosa basedowificata zu bezeichnen ist.

Meine Ausführungen beziehen sich auf die reinen Grundformen der Struma, die natürlich in den verschiedensten Variationen und Kombinationen vorkommen.

\section{B. Hauptteil.}

I. Die das Wachstum des Kropfes beeinflussenden $\mathrm{Kräfte}$.

Die Energie der Expansion des Kropfes ist verschieden, je nach der Art der Struma, um die es sich handelt, und je nach den Prozessen, die sich in der Struma abspielen, wobei zu berücksichtigen bleibt, ob die Expansion allseitig oder auf einzelne Partien beschränkt ist. Die Einflüsse, bzw. Kräfte, die auf die Struma einwirken, sind im wesentlichen dreierlei Art.

An erster Stelle steht der Widerstand der Umgebung, der sie in der Richtung des geringsten Widerstandes wachsen läßt, wie er sich aus den anatomischen Verhältnissen ergibt, nämlich dorthin, wo sich Gewe bs l ü cke n befinden, oder Ge we be von geringerer Konsistenz, als sie die Struma selbst besitzt, vorhanden sind. Dabei kann der Druck der benachbarten Muskeln durch reflektorische Spannung erhöht und so die Ablenkung des Wachstums im Sinne einer flächenhaften Aus- 
breitung gesteigert werden. In späteren Stadien kommt es zu einer Abnahme dieser Wirkung, indem durch ständigen Druck und dauernde Überdehnung eine Atrophie der betreffenden Muskeln eintritt und dadurch der Struma Ausdehnungsmöglichkeiten geschaffen werden, die unter normalen Umständen nicht bestehen.

Es ist selbstverständlich, daß für die Hypertrophie bzw. Atrophie von Muskeln die Schnelligkeit der Dehnung von Bedeutung ist. Bei schnell wachsenden Strumen tritt infolge plötzlicher Überdehnung eine Atrophie der Muskeln ein, während diese bei langsam wachsenden Strumen Zeit finden, zu hypertrophieren.

Zudem ist zu erwähnen, daß bei schnell wachsenden Strumen der Reiz auf die Umgebung mit der Schnelligkeit des Wachstum's zunimmt. Hierdurch kann es zu einer reaktiven Bindegewebswucherung und damit $z \mathrm{u}$ Verwachsungen kommen.

Die zweite, die Kropfausbreitung beeinflussende Kraft ist die eigene Schwere, die je nach Art und Entwicklungsstadium der Struma verschieden ist.

Die dritte ist die Ansaugung durch den negativen Druck im Thorax bei geringem Wachstum nach unten. Sie erreicht im Moment der Inspiration das Maximum, wodurch noch oberhalb der Thoraxapertur befindliche Kröpfe völlig in den Thorax aspiriert werden können.

Abgesehen von diesen endogenen Kräften wird die Wachstumsrichtung der Struma nicht selten durch exogene, artifizielle Momente modifiziert, wie Halsbinde, Kragen, unsachgemäße Behandlung. Diese wirken entweder im Sinne einer A ufhebung oder Unterstützung der Schwerkraft, d. h. sie verdrängen die Struma nach oben oder unten, oder sie bedingen infolge Behinderung des Dickenwachstums eine flächenhafte Ausbreitung oder Tiefenentwicklung.

II. Topographisch-anatomische Verhältnisse des $\mathrm{H}$ alses.

Als Grundlage des Verständnisses der Ausbreitungsverhältnisse des wachsenden Kropfes wollen wir uns zunächst einmal die topographische Anatomie des Halses vergegenwärtigen, unter Beschränkung natürlich auf die für unsere Zwecke in Betracht kommenden Dinge. Daß wir hierbei nichts Neues bringen, liegt 
auf der Hand, doch rechtfertigt sich eine solche Darstellung vollauf damit, daß bisher nirgends die topographischen Verhältnisse vom Standpunkt der anatomischen Ausbreitungsmöglichkeiten des Kropfes und seiner chirurgischen Bedeutung aus beleuchtet worden $\operatorname{sind} \mathbf{1}$ ).

\section{Allgemeines.}

Der Hals, der in der Wirbelsäule seinen Stützpfeiler hat, wird durch eine vom Processus mastoideus zum Akromion gezogene Linie in eine vordere und hintere Abteilung zerlegt. Die hintere solide Abteilung, die Regio nuchae, kommt für unsere Zwecke nicht in Betracht; die vordere Abteilung, die Regio colli sensu strictiore, faßt man am besten als einen zylindrischen, die Halsorgane beherbergenden Hohlraum auf, der ohne scharfe physiologische Grenze in den Brustraum übergeht, in dem einerseits die Brustorgane, die Lungen mit ihren Spitzen, in den Bereich des Halses hinaufragen, andererseits die Organe des Halses kontinuierlich in den Brustraum übergehen. Auf jeden Fall ist bei Würdigung der topographischen Verhältnisse einer Struma eine scharfe Trennung $z$ wischen Hals und Brustnichtin jeder Beziehung praktisch und richtig, wenn man bedenkt, daß die Lunge und somit der negative Druck des „Thorax“ nicht auf diesen beschränkt sind; sondern 2-3 fingerbreit über die Clavicula reichen.

Gleichwohl ist die obere Thoraxapertur als solche insofern als Grenze oder besser gesagt Schranke wichtig, als größere Strumenknoten bei Ausdehnung nach unten oder bei Senkung an ihr einen Widerstand finden. Sie können jedoch auch dann noch einseitig in den Thorax weiter wachsen, wodurch, wenn gleichzeitig ein Wachstum nach außen erfolgt, die reitenden Kröpfe entstehen.

2. Oberflächenanatomie.

Betrachtet man den Hals von vorn, so wird bei kräftiger Muskulatur und mäßigem Fettpolster die Halsregion im engeren

I) Bei meinen anatomischen Untersuchungen unterstützte mich trefflich Herr Wolfgang Boeheimer aus Attendorn in Westfalen. Der junge hoffnungsvolle Kollege wurde der Fortführung und Erweiterung dieser Ergebnisse durch den Tod entrissen. 
Sinne durch den vom Processus mastoideus zur Incisura jugularis sterni und zum sternalen Ende der Clavicula herabziebenden $M$. sternocleidomastoideus in zwei größere Dreiecke geteilt; ein mediales, dessen Basis durch den unteren Rand des Unterkiefers gebildet wird (Trigonum colli mediale), und ein laterales, dessen Basis durch die Clavicula dargestellt wird. Im medialen Dreieck ist der Schildknorpel und mitunter die Schilddrüse als leichte Prominenz zu sehen, im lateralen Dreieck bei starker Anspannung der Halsmuskulatur bisweilen der untere Bauch des vom Zungenbein zur Incisura scapulae ziehenden M. omohyoideus.

3. Tiefenanatomie.

a) Die Halsmuskulatur.

Die Muskulatur des Halses bildet, wenn man den Befund in schematischer Form zusammenfaßt, wie oben erwähnt, einen $\mathrm{Hohlzy}$ inder, in welchem die in lockeres Bindegewebe eingebetteten Halsorgane nebst Gefäß- und Nervenstämmen verlaufen. - Die erste Schicht wird von dem M. sternocleidomastoideus und dem M. trapezius gebildet. Beide Muskeln bilden mit dem oberen Rand der Clavicula das Trigonum colli laterale. Im Bereiche desselben und in einer tieferen Schicht sind von der Seite her und von oben nach unten zu sehen: Die Mm. splenius, levator scapulae, scalenus medius und anterior, welche gewissermaßen den Boden des Trigonum colli mediale bilden, der unten vorn noch von dem unteren Bauch des M. omohyoideus überkreuzt wird.

Die tiefste Muskelschicht der vorderen Halsregion wird durch die $\mathrm{Mm}$. longi colli et capitis dargestellt, welche sich auf der vorderen Fläche der Halswirbelsäule von dem dritten Brustwirbel bis zum Tuberculum pharyngeum des Hinterhauptbeines erstrecken und denen sich seitlich die oben erwähnten Scaleni anschließen. $\mathrm{Da}$, wo die Scaleni zu ihren Insertionen an der ersten Rippe auseinandergehen, bilden sie mit der ersten Rippe die hintere Scalenuslücke, in welcher die Nn. cervicales zur Bildung des Plexus cervicalis und brachialis zusammentreten und durch die die A. subclavia austritt. Der Scalenus anterior bildet mit der ersten Rippe und der Clavicula den Durchtrittsraum für die Vena subclavia, die vordere Scalenuslücke. 
Im vorderen Halsdreieck, speziell in dem für uns in Betracht kommenden Trigonum infrahyoideum, das ist der Teil des vorderen Halsdreiecks unterhalb des Zungenbeins, befinden sich nur die Mm. sternohyoidei und thyreoidei, zwei schwache Muskelpaare, die vom Sternum entspringen und, von der Fascia colli media bedeckt, zum Zungenbein bzw. Schildknorpel ziehen.

\section{b) Die Halsfascien.}

Eine wesentliche Ergänzung erhält der durch die Halsmuskeln gebildete Hohlzylinder durch die Halsfascien, deren drei unterschieden werden: Die Fascia colli superficialis, die Fascia colli media und die Fascia colli profunda. Erstere und letztere sind zu Fascienblättern differenziertes Bindegewebe des Halses, das die Muskulatur einscheidet, während die Fascia colli media sich über den Rang einer gewöhnlichen Muskelfascie erhebt, indem sie aponeurotische Struktur besitzt und auf eine bei niederen Säugetieren ausgebildete, beim Menschen reduzierte oder blo $B$ als Varietät vorhandene Muskelschicht zurückzuführen ist.

Die recht schwache Fascia colli superficialis, die für uns wichtigste Halsfascie, kommt nach Entfernung der subkutanen Fettschicht und des Platysma zur Ansicht. Sie setzt hinten am Lig. nuchae an, scheidet den M. trapezius ein, überdeckt das laterale Halsdreieck und zeigt hier zahlreiche, durch den Durchtritt von Hautnerven und Venen bedingte Lücken, scheidet weiter vorn den $M$. sternocleidomastoideus ein und bildet den Abschluß des vorderen Halsdreiecks.

Die Fascia colli media dehnt sich vom Zungenbein bis zum Sternum zwischen den beiden Mm. omohyoidei aus und ist teils fest mit der Fascia colli superficialis verbunden, teils durch einen schmalen Spalt von lockerem Bindegewebe von ihr getrennt. Sie verleiht der Fascia colli superficialis in dem zentralen Teil des vorderen Halsdreiecks und dem Trigonum colli laterale unterhalb des unteren Omohyoideusbauches eine Verstärkung.

Die Fascia colli profunda sive praevertebralis überzieht die $\mathrm{Mm}$. longi colli et capitis sowie die vordere Fläche der Halswirbelsäule und hängt seitlich mit der die Scaleni ein. schließenden Scheide zusammen. Die Gefäßscheide der Hals- 
gefäße steht ihrerseits wieder in Verbindung nach der einen Richtung mit der Fascie der Scaleni, nach der anderen mit der Fascia colli media, wodurch der Abschluß des Halszylinders vollständił wird.
c) Die Fascienräume.

Durch diese 3 Fascienschichten wird der Hals in drei Fascienräume eingeteilt (Fig. I). Der erste, der schmale Spalt zwischen Fascia colli superficialis und media, ist von geringer praktischer Bedeutung; der dritte, ein osteofibröser Raum, der durch

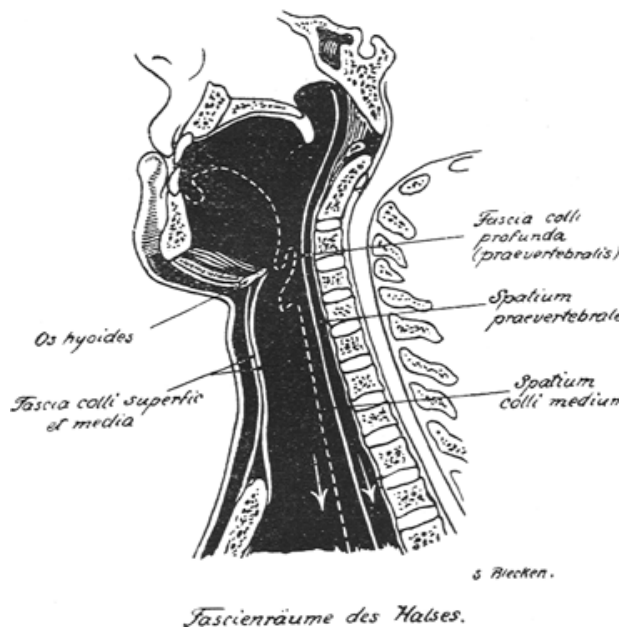

Fig. I.

(Nach Corning).

die Fascia colli profunda und den vorderen Umfang der Halswirbelsäule gebildet wird, kommt für unsere $Z$ wecke nicht in Bctracht, während der mittlere Bindegewebsraum, das S patium colli medium, der für uns wichtigste ist. Er liegt unterhalb des Hyoidknorpels zwischen der Fascia colli media und superficialis einerseits und der Fascia colli profunda andererseits, ist von lockerem Bindegewebe ausgefült, in dem die Halsorgane eingebettet liegen, und geht $n a c h a b w a ̈ r t s$ in den Mediastinalraum, nach aufwärts in das Spatium parapharyngcum und in die Fossa retromandibularis kontinuierlich über, indem die beiden letzten Re- 
gionen als Teile des Spatium colli medium aufgefaßt werden können.

Von großer Wichtigkeit sind die Beziehungen der Lungenspitzen zu den Gebilden des Spatium colli medium, indem der A pex pulmonis beiderseits, nach vorn projiziert, in den $\mathrm{Bereich} \mathrm{der} \mathrm{Halsregion} \mathrm{fällt,} \mathrm{wenn} \mathrm{wir} \mathrm{als} \mathrm{untere} \mathrm{Grenze}$ der letzteren die Incisura sterni jugularis und den oberen Rand der Clavicula annehmen.

d) Die normale Schilddrüse.

Die $30-60 \mathrm{~g}$ wiegende normale Schilddrüse besteht aus zwei seitlichen, der Trachea und den Kehlkopfknorpeln halbmondförmig aufliegenden, längsgestellten Lappen, die durch. schnittlich je 5-8 $\mathrm{cm}$ lang, 3-4 $\mathrm{cm}$ breit und in der Mitte $1,5-2 \mathrm{~cm}$ dick sind. Die Lappen liegen im Mittel in Höhe des 2. bis 3. Trachealringes, treten nach hinten in Beziehung zum Pharynx bzw. Ösophagus, seitlich und hinten zu den Scheiden der großen Halsgefäße und werden vorn von der Fascia colli media überdeckt. Die beiden Seitenlappen besitzen ein schmales Verbindungsstück, den $0,5-1,5 \mathrm{~cm}$ langen Isthmus, der dem 2. bis 3. Trachealring, nicht selten auch dem Krikoidknorpel in seinem vorderen Umfange aufliegt und mit seinem unteren Rand 2,5 bis $3 \mathrm{~cm}$ über der Incisura jugularis sterni steht.

Die Form der Thyreoidea weist, ohne ins Pathologische zu greifen, die verschiedensten Variationen auf, die hauptsächlich den Isthmus betreffen. Hier kann der Processus pyramidalis, der Überrest des Ductus thyreoglossus, der meistens zugrunde geht, mehr oder minder stark ausgebildet sein und bis zur Bildungsstätte der Drüse am Zungengrund, dem Foramen coecum, reichen.

Die Lage des Isthmus hinter der Trachea und hinter dem Ösophagus sind als Entwicklungsanomalien anzusehen.

Die Schilddrüse besitzt zwei Kapseln, die Capsula interna und externa. Während erstere als echte Drüsenkapsel im Zusammenhang mit dem bindegewebigen Gerüst der Drüse steht, ist letztere ein Derivat des Halsbindegewebes und stellt eine recht derbe Kapsel dar, die durch ihre Verbindung mit der Trachea, der Cartilago cricoidea und den Fascien der Mm. sternothyreoidei und thyoidei die Fixation der Drüse bewirkt. 
III. Die Ausbreitungsmöglichkeiten des Kropfes. Wenden wir uns nun den Ausbreitungsmöglichkeiten der Struma im allgemeinen und besonderen zu.

I. Allgemeine Ausbreitungsmöglichkeiten.

Man kann grob schematisch ein Wachstum der Struma nach oben, nach unten und eine Ausdehnung in horizontal-radiärer Richtung unterscheiden. Während bei dem Wachstum nach oben die Struma ungehindert durch das Spatium parapharyngeum bis in die Fossa retromandibularis gelangen kann, setzen der nach unten wachsenden Struma nach Überwindung der oberen Thoraxapertur erst die Organe des Mediastinums bzw. das Zwerchfell ein Hindernis entgegen.

Anders beim Wachstum der Struma in horizontal-radiärer Richtung. Hier müssen wir zwei die Ausdehnung beeinflussende Komponenten unterscheiden: Erstens die Ge we bs l ü cken bzw. Muskellücken weisen der wachsenden Struma den Weg, zweitens die die Lücken verschließenden Gewebe, die Fascien, setzen den Ausbreitungsmöglichkeiten eine Schranke, während der Widerstand, der der einseitig wachsenden Struma durch die Halsorgane gesetzt wird, unberücksichtigt bleiben kann, da dieselben infolge ihrer lockeren Verbindung mit der Umgebung weitgehende Dislokationen ermöglichen.

2. Die speziellen Ausbreitungsmöglichkeiten des Kropfes.

a) In horizontal-radiärer Richtung.

Bei der häufigsten Ausdehnung der Struma in horizontalradiärer Richtung findet sie an der Halswirbelsäule mit ihrer kompakten Muskulatur ein unüberwindliches Hindernis, während sie seitlich und vorn die Fascia colli superficialis bzw. media nur an den Stellen erreichen kann, wo der darunter gelegene Muskelzylinder Lücken aufweist, die rein theoretisch folgende sind:

I. Hintere Scalenuslücke,

2. vordere Scalenuslücke,

3. die Lücke zwischen dem vorderen Rand des Scalenus 
anterior und dem oberflächlicher liegenden M. sternocleidomastoideus,

4. der Raum zwischen den beiden Mm. sternocleidomastoidei, wenn man die äußerst schwachen vorderen Halsmuskeln unberücksichtigt läßt.

Betrachtet man den Halssitus von der Seite, so sieht man, daß der laterale Rand der Thyreoidea mit dem vorderen Rand des Scalenus anterior fast in einer Ebene liegt, so daß die Thyreoidea nach hinten und seitlich wachsen $m u B$, falls die vordere oder hintere Scalenuslücke als Durchtrittsraum für sie in Betracht kommen sollen. Da nun diese beiden Aperturen, besonders erstere, durchaus in keinem Verhältnis stehen zu der Größe eines Kropfes, wenn er bis zu ihnen vorgedrungen ist, so kommt es weniger zu einem Durchtritt, vielmehr wird - dies gilt für die hintere Scalenuslücke - durch den Druck der Struma a uf die Muskulatur dieselbe überdehnt, so daß die bis dahin zum Teil verschlossenen $L \ddot{u} \mathrm{ck}$ en $\mathrm{klaffen}$. Jedoch wird sie auch dann noch für eine Struma, die eine derartige Größe erreicht hat, zu klein, um sie durchtreten zu lassen. Sie schmiegt sich vielmehr der nach außen vorgebuchteten Muskulatur an und liegt hier wie in einer Nische, deren Boden durch die obere Thoraxapertur und die durch die Halsmuskellücken austretenden Nerven- und Gefäßstämme gebildet wird. Ein derart festliegender Kropf wird, wenn er sich weiter ausdehnt, energischeren Widerstand in der Peripherie des Halses an den Muskeln und Nervenbündeln finden, als an seiner medialen Fläche, wo die in lockeres Bindegewebe eingelagerten Halsorgane weitgehende Dislokationen ermöglichen. Er wird sich also bei weiterem Wachstum immer mehrmedial schieben.

Wächst ein Strumaknoten rein seitlich, so schiebt er das Gefäßbündel des Halses beiseite und gleitet unter dem Kopfnicker durch die Lücke zwischen letzterem und dem Scalenus anterior in das seitliche Halsdreieck, wo er mühelos die Fascia colli superficialis, den einzigen noch vorhandenen Widerstand, überdehnt und sich dann ungehindert a u sbreiten kann.

Wesentlich einfacher liegen die Verhältnisse bei A usdeh nung der Struma nach vorn, wo das vordere Halsdreieck 
nur von Fascien und schwachen Muskeln ausgefüllt wird. Hier kann die Struma, wenn sie die Gebilde einmal überdehnt hat, sich ungehindert ausbreiten, ohne außer der kosmetischen Verunstaltung irgendwelche Erscheinungen $\mathrm{zu}$ machen.

Falls es sich um einen strumös entarteten Processus pyramidalis handelt, hängen die Erscheinungen davon ab, wie weit der Processus pyramidalis nach oben reicht und wo der Kropfknoten sich ausgebildet hat. Die unterhalb des Hyoidknorpels liegenden Strumenknoten bieten keine Besonderheițen, während die oberhalb und in der Zunge liegenden $\mathrm{zu}$ den aus anormalen Anlagen entstehenden Strumen gerechnet werden müssen und in ihrer Ausbreitung anderen gutartigen Tumoren dieser Region gleichkommen. Im großen und ganzen spielt es keine Rolle, in welchem Teile der Schilddrüse ein Knoten sich ausbildet, sondern lediglich, wohin er wächst, wobei jedoch zu beachten ist, daß die Knoten sich fast immer in den unteren Partien der Seitenlappen, und $z$ war beiderseits gleich häufig entwickeln, um allmählich nach oben fortzuschreiten. Hierbei ist der am tiefsten stehende linke Pol wegen der Gefahr des intrathorakalen Wachstums besonders zu beachten.

b) Das Wachstum nach oben.

Die nach oben wachsende Struma gelangt ohne Widerstand in das Spatium parapharyngeum und weiter in die Fossa retromandibularis und kann bei gleichzeitigem Wachstum nach hinten ungehindert in das $S$ patium retropharyng e um gelangen. Die Schädelbas is bietet erst der Ausbreitung nach oben Einhalt.

Bei gleichzeitigem Dickenwachstum bietet die nach oben wachsende Struma insofern keine Besonderheiten, als sich hierbei die gleichen Vorgänge, die oben beschrieben sind, abspielen, indem allgemein nicht das Wachstum nach oben der ausschlaggebende Faktor für die Erscheinungen, die ausgelöst werden, ist, sondern das Dickenwachstum.

c) Das Wachstum nachunten.

Bej schon geringem Wachstum seitlich und nach untert tritt die Struma in Beziehung.zur Lungenspitze, 
und zwar noch im Bereich des Halses, da, wie oben beschrieben, der Apex pulmonis nach vorn projiziert 2-3 Querfinger breit über den oberen Rand der Clavicula hinaufreicht, und so die oberste Partie der Lungenspitzen in gleicher Höhe mit dem unteren Pol der normalen Thyreoidea zu liegen kommt. Hat die Struma die Gewebe bis z u r Ple u r a verdrängt, so fällt nicht nur an der Stelle, wo sie die Pleura berührt, jeder Widerstand fort. vielmehr unterliegt sie obendrein der Einwirkung des negativen Druckes im Thorax, der eine aktive Ansaugung auf die betreffende Partie der Struma ausübt. Als weiteres Moment ist die eigene Schwere hinzuzufügen, die ihren vollen Einfluß auf den die Pleura berührenden Teil der Struma entfaltet.

Bei weiterer Ausdehnung der Struma nach unten stülpt sich die Ple ura e in und dehnt sich, da sie sich bei ihrer festen Verbindung mit der Umgebung wenig leicht verschieben kann, aus. Auf diese Weise nimmt sie allmählich die Struma wie in einem Beutel auf. Die Lunge wird hierbei zunächst nicht komprimiert, sondern $\mathrm{zieht} \mathrm{sich}$, da sie sich in einem Zustand von Überdehnung befindet, aktiv zusammen, um erst bei äußerster Größenzunahme der Struma passiv komprimiert zu werden. Die Verbindung der Struma mit dem Mutterorgan wird, falls dieses nicht selbst mit herabgezogen wird, stielartig ausgezogen und von der Nachbarschaft zugleich komprimiert, so daß häufig nur ein dünner, oft nur mikroskopisch nachweisbarer Gewebsstreifen übrig bleibt.

Diese Knoten, die mit dem Mutterorgan mit einem dünnen Gewebsstreifen in Verbindung stehen, werden auch $\mathrm{falsche}$ Nebenkröpfe genannt, im Gegensatz zu den echten. Letztere stellen strumös entartete abgesprengte Keime der Schilddrüse dar, die in der Lunge, der Trachea und sogar in der Aorta und im Herzen liegen können.

Hier ist noch zu erwähnen, daB am leichtesten die Struma nicht vor, sondern hinter den großen Gefä Ben die Pleurakuppel erreichen kann, da die Gefäße dieselbe in ihrem vorderen Teile überziehen und so der vordere Durchtrittsraum erstens kleiner und zweitens für die wachsende Struma infolge seiner Lage schwerer zu erreichen ist.

Die in der Medianlinie nach unten in das hintere Media- 
stinum wachsende Struma kann ohne sonderlichen Widerstand bis zum Zwerchfell gelangen, falls sie nicht auf einen Bronchus stößt und auf diesem reitend zu beiden Seiten desselben weiterwächst. Anders ist es dagegen bei der ins vordere Mediastinum wachsenden. Hier findet sie zunächst auch keinen Widerstand, um dann auf ein Hindernis zu stoßen, das nicht überwunden wird, und zwar auf das Herz, dem sich die wachsende Struma allmählich haubenartig auflegt.

In der Praxis sind bis jetzt keinerlei Fälle beschrieben worden, in denen die Struma bei rein medianem Wachstum nach unten vor dem Herzbeutel das Zwerchfell erreicht hätte. Eine Erklärung dafür habe ich in der Literatur nicht gefunden.

Sie liegt einmal in der Einspannung der Struma in den zum Hilus gerichteten Zug der Lungen, der die Senkungstendenz derselben bedeutend verringert. Sodann findet die Struma an dem Herzen als solchem einen Widerstand, der außer der reinen Gewebsfestigkeit des Organs durch seine Kontraktion bedeutend vermehrt wird. Auf diese Weise gesellt sich zu dem reinen Widerstand der Masse des Herzens infolge seiner rhythmischen Kontraktion eine Kraft, die als aktive hämmernde Gegenbewegung eine lebende Energie darstellt und gemäß den physikalischen Gesetzen bis tief in die Struma hineingetragen wird. Hierdurch werden auch die tiefer liegenden Schichten verhindert, in Richtung des Herzens zu wachsen.

Zudem kann sich eine Struma in einer derartigen Lage ungehindert nach allen anderen Richtungen verbreitern, zumal noch der Zug der Lungen außer an der Berührungsstelle mit dem Herzen bzw. Perikard einen gleichmäßigen dauernden Reiz auf die ganze Oberfläche ausübt. Das Perikard spielt hier keine Rolle, da es genau so, wie oben für die Pleura beschrieben, sich ausbuchten würde.

Zum Schluß seien noch die aus Anlageanomalien entstehenden retrotrachealen und retrovisceralen Strumen erwähnt, die aus einem hinter der Trachea bzw. hinter dem Ösophagus gelegenen Isthmus entstehen und in bezug auf ihre Ausbreitung keine Besonderheiten aufweisen. 
IV. Bezeichnung der Kropflagen.

In der Literatur bezeichnet man bisher nach Wölfle r sämtliche nicht an dem normalen Sitz der Schilddrüse liegenden Kröpfe als falsche Nebenkröpfe, wenn sie mit der Thyreoidea in Verbindung standen. Man unterscheidet obere, untere, hintere, seitliche und vordere Nebenkröpfe, die unteren teilte man wieder ein in substernale, endothorakale und retroclaviculäre Nebenkröpfe.

Diese Bezeichnungen sind sehr dehnbar und lassen das Charakterstikum einer Kropflage ganz außer acht, indem z. B. eine Struma retroclavicularis nach den vorigen Ausführungen sowohl innerhalb des Brustraums, medial und seitlich der Scaleni und im seitlichen Halsdreieck liegen kann. Hieraus ersieht man, wie wesentlich eine präzisere Bezeichnung der einzelnen Strumalagerungen ist.

Die nach oben in das Spatium parapharyngeum und in die Fossa retromandibularis wachsenden Kröpfe bezeichnet man am besten nach ihrem Sitz als Struma parapharyngeae und retromandibulares, die in den Retropharyngealraum wachsenden als Strumae retropharyngeae.

Die medial von den Scaleni nach hinten und seitlich wachsenden Kröpfe, die als Charakteristikum ihre laterale Fixation an den Scaleni aufweisen und bei Größenzunahme sich unter Dislokation der Trachea immer mehr medial schieben, werden durch die Bezeichnung charakterisiert: Strumae colli laterales posteriores sive fixatae; die nach vorn seitlich zwischen Scalenus anterior und Sternocleidomastoideus ins seitliche Halsdreieck wachsenden Kröpfe, die sich hier ungehindert ausbreiten können, nennt man am besten Strumae colli laterales sive liberae, die zwischen den beiden Sternocleidomastoidei nach vorn wachsenden Strumen als Strumae colli anteriores.

Die nach unten seitlich wachsenden Kröpfe, die als Charakteristikum aufweisen, daß sie allmählich von der Pleura wie in einem Beutel aufgenommen werden, bezeichnet man treffend als Strumae intrapleurales.

Die ins vordere und hintere Mediastinum wachsenden Kröpfe zeigen keine spezifische Besonderheiten und werden daher als Strumae mediastinales anteriores und posteriores genügend charakterisiert. 
V. Die Symptomatologie der verschiedenen Strumen.

I. Die Strumen des Halses.

Die Abgrenzung der durch mechanische Einflüsse hervorgerufenen Symptomenkomplexe, mithin die genaue Kenntnis der Topographie ist deshalb von so großer fraktischer Bedeutung, weil häufig die Ausbreitung des Kropfes ịn einer bestimmten Richtung nur an Funktionsstörungen von Organen, Nerven usw. erkannt wird, deren topographische Lage bekannt sein muß, wenn man von den Störungen umgekehrt wieder auf die Lage der Struma schließen will. Man könnte hier einwenden, daß bei unsicheren Fällen heutzutage stets Röntgenbilder gemacht werden. Zur Deutung der Röntgenbilder ist jedoch die genaue Kenntnis der Topographie ebenso erforderlich, da erstens sehr oft die Struma nur durch Verlagerung sichtbarer Gebilde, also indirekt erkannt wird, zweitens eine genaue Kenntnis der Verhältnisse des Skelettsystems zur Rekonstruktion eines plastischen Bildes aus der Röntgenplatte unbedingtes Erfordernis ist. Als Beweis dafür, wie wichtig die Topographie ist und welche Trugschlüsse durch Vernachlässigung obiger Ausführungen herbeigeführt werden, zeigt folgendes Beispiel, indem sowohl die klinischen Erscheinungen, als auch das Röntgenbild nicht richtig gedeutet wurden.

Fall I. Sarbo, Zur Symptomatologie der intrathor a kischen Struma. Wiener klin. Wochenschr., Bd. 26, II., S. 1979 .

Aus der Vorgeschichte: Seit $3^{1 / 2}$ J. Schreibkrampf. Vor $2^{1 / 2} \mathrm{~J}$. wurden $\mathrm{P}$. die Kleidungsstücke in der rechten Achselhöhle zu eng, ohne daß er fetter geworden wäre, gleichzeitig Schmerzen im rechten Arm und in der rechten Schulter, die ,immerfort auftraten". Starkes ständiges Schwitzen, besonders in rechter Achselhöhle. Seit Beginn des Leidens Konsultation von vielen Spezialisten, die Neuralgie, Neurasthenie, Rheumatismus usw. diagnostizierten. Aus dem Status: Typische Basedowsymptome, rechtseitig vergrößerte Schilddrüse, Druckempfindlichkeit des I. und 2. Dorsalwirbels.

$\mathrm{Da}$ Verfasser eine intrathorakische Struma vermutete, ließ er folgendes Röntgenbild machen (Fig. 2).

Diesem Röntgenbild gibt er als Ergänzung nachstehenden Text:

„Die Abbildung zeigt in deutlicher Weise, wie stark die Trachea nach links verlagert war. Außerdem sieht man an der linken Seite des 5 . bis 7 . Halswirbels eine vertikal verlaufende, $4 \mathrm{~mm}$ breite, vollkommen verkalkte Arterie, welche wohl der A. vertebralis ent- 
sprechen wird; an der rechten Seite sieht man anderseits am Wirbelkörper des 6 . Halswirbels eine schräg verlaufende, andere verkalkte Arterie, wahrscheinlich die A. thyreoidea." Operation (durch anderen Arzt):

„Der rechte Lappen wurde subkapsulär fast in seinem ganzen Umfang entfernt, nur der an den Isthmus angrenzende Teil des Lappens blieb drinnen. Dieser Teil mochte $1 / 6$ des ganzen Lappens ausmachen."

Weiter schreibt Verfasser : „Der entfernte Tumor war faustgroß.“

Nach Aufzählung der für eine Struma intrathoracica charak. teristischen Symptome sagt er zum Schluß:

„Wie ersichtlich, finden wir die unseren Fall beherrschenden Symptome, das profuse Schwitzen in der Achselhöhle und dadurch bedingte neuralgische Schmerzen, den Schreibkrampf, nicht erwähnt;

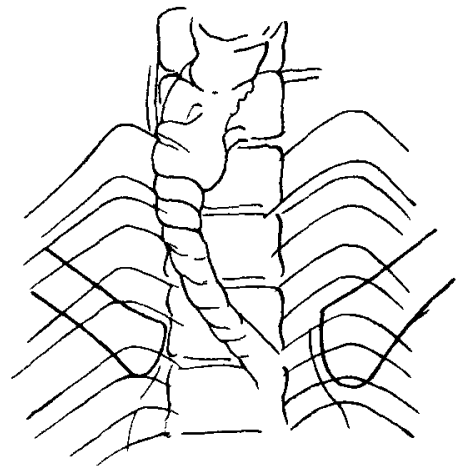

Fig. 2.

Röntgenbild zu Fall $\mathbf{~}$.

anderseits waren in meinem Fall die .oben angeführten Symptome (einer intrathorakischen Struma) zum großen Teil nicht vorhanden oder nur sehr wenig ausgeprägt."

„... Den größten Dienst hat in diagnostischer Hinsicht die Röntgendurchleuchtung geleistet."

In obiger Abhandlung ist erstens nicht erwähnt, wie Verfasser zu der Diagnose Struma intrathoracica bzw. substernalis auf Grund der Symptome und vor allem des Röntgenbildes gekommen ist, zweitens fehlt im Operationsbericht, wo eine Struma entfernt wurde. In vorliegendem Falle lassen die diagnostischen Erwägungen hinsichtlich des Sitzes der Struma bei richtiger Bewertung der klinischen Symptome und des Röntgenbildes keinerlei Zweifel über den Sitz des Kropfes zu, allerdings ist Voraussetzung die genaue Kenntnis der topographischen Verhältnisse. 
Deutung der klinischen Erscheinungen:

I. Der Schreibkrampf wurde offensichtlich durch Druck der Struma auf die den Arm versorgenden Nerven hervorgerufen. Der Arm wird vom Plexus brachialis innerviert, der von den Rami anteriores der Nn. cervicales V-VIII und vom N. thoracalis I gebildet wird, indem die Muskeln des Armes und der Schulter speziell vom 5. und 6. Cervikalsegment versorgt werden. Da der Plexus brachialis der Struma nur innerhalb des Halszylinders zugänglich ist, und zwar in einem Dreieck, dessen Basis vom 5. Hals- bis zum 6. Brustwirbel reicht und dessen Spitze im unteren Teil der hinteren Scalenuslücke liegt, so muß sie innerhalb dieses Dreiecks auf den Plexus gedrückt haben, d. h. die Struma muß innerhalb des Halszylinders medial von der Scaleni nach hinten gewachsen sein.

2. Die neuralgischen Schmerzen in Arm und Schulter sind auf die gleiche Weise zu erklären, da der Plexus brachialis Schulter und freie Extremität sensibel versorgt.

3. Das abnorm starke Schwitzen in der rechten Achselhöhle war, falls es sich nicht um ein thyreotoxisches Moment handelte, durch mechanische Sympathikusreizung in derselben Weise hervorgerufen. Dafur, daß der Grenzstrang direkt nicht in Mitleidenschaft gezogen wurde, d. h. daß die Struma nicht auf die hintere Wand des Halszylinders drückte, spricht der Umstand, daß keinerlei typische Reizerscheinungen des Halssympathikus vorlagen.

4. Die Druckempfindlichkeit des I. und 2. Dorsalwirbels ist wegen des eben erwähnten Fehlens von Reizerscheinungen des Halsgrenzstrangs nicht auf direkte Läsion der Wirbelkörper zurückzuführen, es wurde vielmehr durch den dauernden Reiz, der auf den Plexus durch die Struma ausgeübt wurde, eine Übererregbarkeit der zu dem Plexus gehörigen Rückenmarkssegmente herbeigeführt. Da der Arm und die Schulter von dem 5. und 6. Cervikalsegment versorgt wird, so tritt nach den Forschungen von $\mathrm{H}$ e a d durch anhaltende Reizung der zugehörigen zentripetalen Fasern eine Übererregbarkeit dieser beiden Segmente und damit eine Hyperästhesie der von ihnen sensibel versorgten Zonen ein. Nun entspricht eine Hyperästhesie im Bereiche des I. und II. Thorakalwirbels nach $\mathrm{H}$ ead einer Reizung des 5. und 6. Cervikalsegments. Diese Segmente versorgen, wie anfänglich erwähnt, die Schulter und den Arm. Es folgt hieraus, daß durch die Reizung des Armplexus das 5. und 6. Cervikalsegment übererregt war, wodurch eine Hyperästhesie in der Gegend des I. und 2. Thorakalwirbels resultierte. Oder umgekehrt spricht die Hyperästhesie in diesem Falle für eine Läsion der von dem 5. und 6. Cervikalsegment ausgehenden Nerven, also der Armnerven, die, wie oben erwähnt, im Bereiche des Halszylinders statthatte.

Die klinischen Erscheinungen, der Schreibkrampf, die neuralgischen Schmerzen in Arm und Schulter rechts, die abnorme 
Schweißsekretion in der rechten Achseldrüse, die Druckempfindlichkeit des I. und II. Thorakalwirbels sprechen für eine Struma, die im Bereiche des Halszylinders medial von den Scaleni nach hinten gewachsen war, da sie nur hier den Plexus erreichen konnte. Es handelte sich demnach um eine Struma colli posterior fixata (s. Theoret. Teil).

Die Verlagerung der Trachea war dadurch bedingt, daß die Struma lateral an den Scaleni einen bedeutend größeren Widerstand fand, als medial an den locker fixierten Halsorganen. Sie hatte sich daher bei weiterer Dickenzunahme immer mehr medial geschoben. Ich habe hier bei der Deutung des beiliegenden Röntgenbildes die Verbiegung der Trachea oberhalb des Brustkorbes vorausgesetzt.

Deutung des Röntgenbildes.

Nach dem Röntgenbild liegt keine Veranlassung vor anzunehmen. daß eine Struma intrathoracalis bestanden hat. Die einzige Möglichkeit auf eine Struma zu schließen, gibt die Dislokation der Trachea. Da diese Verbiegung im Röntgenbild einerseits unterhalb des 7. Brustwirbels liegt, woraus man bei oberflächlicher Betrachtung die Diagnose Struma intrathoracica stellen könnte, andererseits sich aber oberhalb des Sternums und des sternalen Endes der Clavicula sich befindet, muß man sich zunächst über die räumlichen Beziehungen, d. h. über die Überschneidungsverhältnisse der sichtbarben Gebilde und somit über die Richtung des Ganges der Röntgenstrahlen und die Lage der aufnehmenden Platte Klarheit verschaffen.

Ich habe dem beiliegenden Bild entsprechend die Verhältnisse folgendermaßen rekonstruiert (Fig. 3):

Der Ausgangspunkt der Strahlen liegt dorsal in der Gegend des 3. bis 4. Brustwirbels. Die Strahlen sind dann schräg aufwärts gegangen, und zwar vom 4 . Brustwirbel ungefähr zum sternalen Ende der Clavicula, vom 7. Halswirbel zum Ringknorpel, so daß bildgerecht der obere Rand des Sternums auf den 4 . Brustwirbel, der Ringknorpel auf den 7. Halswirbel zu liegen gekommen ist. Es ist also zweifelsohne die Partie der Trachea oberhalb des Sternums seitlich ausgebuchtet, die Partie der Trachea hinter dem Sternum ist nicht zu sehen. Wenn man bedenkt, daß der 
entfernte Tumor faustgroß war, so muß er genau in die ausgebuchtete Partie der Trachea hineingepaßt haben und konnte höchstens mit seinem unteren Pol in die Thoraxapertur hineinragen. Das Röntgenbild beweist also die Richtigkeit der klinischen Diagnose.

Fall 2. Struma colli lateralis posterior sivefixata. Wuhrmann: Die Strumaintrathoracica. Deutsche Zeitschr. f. Chir., Bd. 43. Mitgeteilt von Proust.

Aus der Vorgeschichte: Beim ersten Spitalaufenthalt wenig entwickelter Tumor an der linken Halsseite, dagegen anormale Entwicklung der Venen auf Vorderseite des Halses und der Brust. Seit Io Monaten Zunahme des Volumens des Tumors und der Venendilatation.

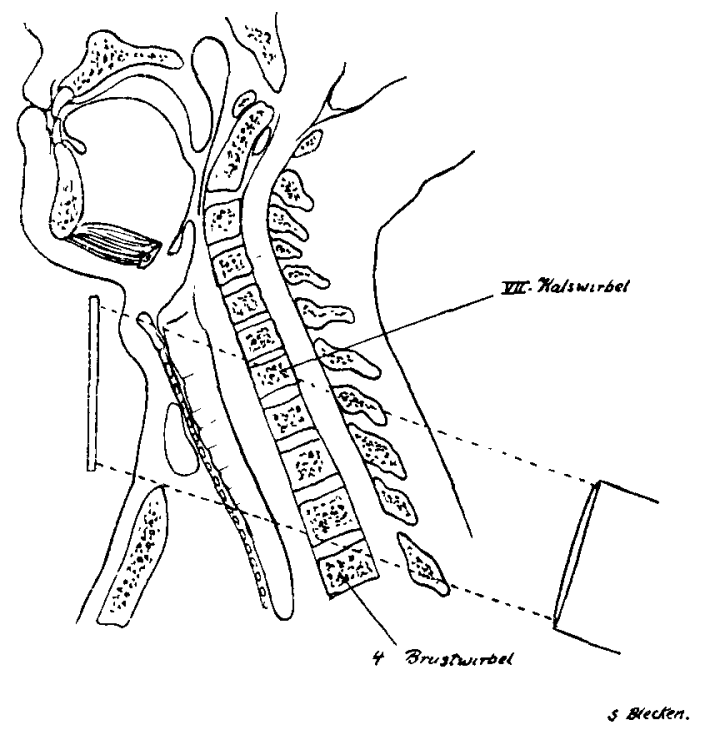

Fig. 3.

Fall I. Rekonstruktion der Röntgenaufnahme.

Aus dem Status: Tumor liegt unter dem Sternocleidomastoideus und ist von ovaler Form. Ungefähre Grenzen: Nach oben zweifingerbreit unter dem Unterkiefer, rechterseits Trachea, die beträchtlich nach links abgewichen. „In der Tiefe, d. h. nach hinten, scheint sich der Tumor an die Wirbelsäule anzustemmen. Nach unten taucht er hinter die Clavicula und kann durch Palpation nicht genau be. stimmt werden."

„P. bemerkte, daß er leicht außer Atem kam, in der Nacht hat er oft Alpdrücken, und dabei weckt ihn dann immer das Gefühl, als ob man ihm die Kehle zuschnüre. Vielleicht trägt dazu die Gewohnheit des Kranken bei, täglich 21 Wein und I l Kaffee zu trinken." 
Enorme Venendilatation der Haut, Herausgeschleudertwerden des Kropfes durch Hustenstöße, gleichzeitige Turgeszenz der Haut, des Gesichts und des Halses. Kein Stridor. Druck auf den Tumor ruft „Hustenkrampf" hervor. Fingerexploration des Rachens o. B.

Ungleichheit der Pupillen.

„Gegenwärtig besteht kein Ödem des Gesichts und des linken Armes, dagegen versichert der Kranke, daß es bisweilen an beiden Orten erscheine."

Anormale Vorwölbung in Höhe des 6. Cervikalwirbels, kein Schmerz daselbst. Dagegen Druckempfindlichkeit des 3 . und 4. Cervikalwirbels. Niemals Neuralgien dieser Gegend.

Im linken Interskapularraum rechts deutliche Dämpfung. Linkerseits nach innen von der Scapula Bronchialatmen. Respiration im Oberlappen unvollständig, Rasselgeräusche. Exitus letalis bei gleichem Lokalbefund und Verschlimmerung des Allgemeinzustandes. Bedeutende Schwellung der linken Gesichtshälfte.

Aus dem Sektionsbefund: Der Sternocleidomastoideus ist nach außen verdrängt. Der Tumor ist rund, steigt hinter der linken Articulatio sternoclavicularis in den Thorax hinab bis zum 2. I.-K.-R. Nervus phrenicus nach außen verdrängt, sonst intakt. Sympathicus zwischen Tumor und Wirbelsäule eingeklemmt, nicht atrophiert. Tumor ist faustgro $B$ und haftet ohne Stiel der Trachea an. Trachea ist bedeutend verdrängt, aber nicht verengt. Herz normal. Die Lungen zeigen an ihrer Spitze mehr konfluierende, in den übrigen Teilen mehr zerstreute tuberkulöse Infiltrationen. Linkerseits besteht eine ungefähr eigroße Kaverne, rechts mehrere kleinere. Von einer Veränderung der Halswirbelsäule ist nirgends die Rede.

Die Symptome dieses Falles und ihre Bewertung für den Sitz der Struma sind um so mehr einer eindeutigen Erklärung zugänglich, als der Sektionsbericht eine Kontrolle zuläßt.

Die Ungleichheit der Pupillen ist auf Reizung oder Lähmung des Sympathicus zurückzuführen, wodurch der Beweis gegeben ist, daß der Tumor gegen die hintere Wand des Halsmuskelzylinders sich anstemmte. $\mathrm{Ob}$ es sich im vorliegenden Falle um eine Reizung oder Lähmung gehandelt hat, läßt sich aus dem Status nicht ersehen, da unerwähnt geblieben ist, ob die Pupille der kranken oder gesunden Seite größer war und welche von beiden Pupillen ev. auf direkten Lichteinfall nicht mehr reagierte.

Die okulopupillären Phänomene bei Lähmung des Halssympathicus sind Verengerung der Pupille, Verkleinerung der Lidspalte und Zurückweichen des Bulbus, bei Reizung Erweite- 
rung der Pupille, Vergrößerung der Lidspalte und Prominenz des Bulbus. Zudem zeitigt Lähmung des Halssympathicus Erlöschen der Pupillenreaktion auf direkten Lichteinfall.

Die anamnestische Angabe des Kranken, daß3 zeitweilig Ödeme des Gesichts und des Armes bestanden haben, ist vielleicht auch auf Sympathicusläsion zurückzuführen, indem man bei Lähmung des Halssympathicus Röte und Hitze, bei Reizung Blässe und Kälte der gleichseitigen Gesichtshälfte beobachtete; ebenso sind Ödeme bei Sympathicusreizung in der Literatur beschrieben. Wahrscheinlich handelt es sich jedoch um eine passive Hyperämie infolge mechanischer Behinderung des venösen Abflusses durch Kompression der Venen von seiten der Struma, da gleichzeitig eine enorme Venendilatation des Halses und der oberen Brust vorlag.

Die Tatsache, daß der Tumor bei Hustenstößen in die Höhe geschleudert wurde, beweist, daß er mit dem Brustraum in Verbindung stand, indem der bei Hustenstößen entstehende Überdruck dem Tumor mitgeteilt wurde; sie beweist jedoch nicht, daß der Tumor die obere Thoraxapertur überschritten hat, da die Lunge $2-3$ Finger breit über die Clavicula reicht. Allerdings traf dies nach dem Sektionsbericht zu. Man sieht hieraus, daß eine scharfe Trennung zwischen Hals und Brust nicht immer gut einzuhalten ist.

Den „Hustenkrampf“, der durch Druck auf den Tumor ausgelöst werden konnte, kann man nicht durch Kompression der Lunge erklären, da bei der großen Relaxionsfähigkeit der Lunge eine derart geringe Abnahme des Pleuraraumes keinen Reiz auf sie auslösen kann. Zerrungen der Lunge infolge Verwachsungen sind nach dem Sektionsbericht ausgeschlossen. Zudem gibt der Ausdruck „Hustenkrampf“" und die Bemerkung im Sektionsbericht, $\mathrm{daB}$ der Phrenikus verlagert sei, ferner das Alpdrücken und die Druckempfindlichkeit des 3. und 4. Cervikalwirbels eine andere eindeutige Erklärung.

Die Tatsache, daß der Phrenikus nach außen verdrängt war, zeigt, daß der jetzige Weg, den der Phrenikus zurücklegen mußte, größer als der normale war, daß also der Nerv unter einer Spannung stand, die bei weiterer Verlagerung als Zerrung auf sein zugehöriges Ganglion übertragen wurde. Durch Druck auf den 
Tumor entstand infolge der seitlichen Expansion desselben eine Anspannung des Phrenikus und Zerrung des zugehörigen Ganglion, das den Reiz weiter zu dem zugehörigen 4. Cervikalsegment weiterleitete. Nun sendet das 4. Cervikalganglion, durch die zentripetalen Impulse gereizt, einmal einen motorischen Impuls durch den Phrenikus zum Zwerchfell, der durch den direkten Reiz des Nerven noch verstärkt wird. Auf diese Weise erklärt sich der „Hustenkrampf" durch Zwerchfellkontraktionen. Anderseits gehen die sensiblen Bahnen des 4. Zervikalsegments dorsal in die Gegend des 3. und 4. Cervikalwirbels (nach $\mathrm{Head}$ ). Diese Region war nach der Krankengesichte druckempfindlich. Genau so gut kann man jetzt umgekehrt sagen: Die Druckempfindlichkeit des 3. und 4. Cervikalwirbels ist ein Symptom für eine Läsion des Phrenikus. Das Alpdrücken usw., das Verfasser durch Koffeinund Alkoholabusus zu erklären sucht, ist ebenfalls aus den durch Phrenikusläsion entstandenen Zwerchfellkontraktionen zu erklären. Wie weit eine direkte Kompression des Phrenikus mitgewirkt hat, läßt sich nicht nachweisen.

Die Vorwölbung in der Gegend des Processus spinosus 6 ist diagnostisch nicht zu verwerten, da sie häufig und physiologisch ist, zumal der Sektionsbericht keine Veränderung der Wirbelsäule erwähnt.

Die Dämpfung und die auskultatorischen Phänomene ergeben sich aus den tuberkulösen Veränderungen der Lunge.

In vorliegendem Falle beweist die Pupillendifferenz, daß die Struma die hintere Wand des Halszylinders komprimierte, also medial von den Scaleni nach hinten gewachsen war, die Venektasien auf Hals und Brust, das zeitweise Ödem des Armes und des Gesichts beweist die Erschwerung des venösen Abflusses; die Druckempfindlichkeit des 3. und 4. Cervikalwirbels, die Hustenkrämpfe bei Druck auf den Tumor, das Alpdrücken sprechen für eine Läsion des Phrenikus, also für Ausbreitung des Tumors über den vorderen Rand des Scalenus, auf dem der Phrenikus liegt, lateral in das seitliche Halsdreieck. Die Verlagerung der Trachea war genau wie im ersten Falle bedingt.

Dieser Fall wurde von $\mathrm{Wuhrmann}$ als Struma intrathoracica angesprochen, wenngleich sämtliche klinischen Erscheinungen keinen Anhaltspunkt dafür bieten. Daß der untere Pol bis zum 
2. I.-K.-R. reichte, geht aus dem Sektionsbericht hervor. Jedoch machte er keine Erscheinungen. Die klinischen Erscheinungen waren sämtlich von einer Struma colli lat. posterior s. fixata ausgegangen. Die höher seitlich des Pharynx sitzende Struma, die Struma parapharyngea, entspricht bei Wachstum nach seitlich hinten den oben beschriebenen Fällen, ebenso die Struma retromandibularis. Eine hierbei vorkommende Läsion des Hypoglossus gehört in das Gebiet der Raritäten. Die Struma retropharyngea macht dieselben Erscheinungen wie ein Retropharyngealabszeß. Die unter dem Kopfnicker in das seitliche Halsdreieck und die zwischen den beiden Kopfnickern nach vorn wachsenden Strumen, die Struma colli lat. ant. s. libera et Struma colli anterior machen außer der kosmetischen Verunstaltung keine klinischen Erscheinungen, abgesehen davon, daß die ins seitliche Halsdreieck wachsende Struma unter Umständen wie in Fall 2 den Phrenikus in Mitleidenschaft ziehen kann. Die bei ringförmigem Wachstum des Kropfes und doppelseitigem Wachstum nach hinten vorkommenden Tracheaerweichungen und Rekurrensläsionen sind zur Genüge bekannt und beschrieben. Es seien hier nur kurz die beiden Arten des Kropftodes erwähnt, der einmal durch Kollabieren der Trachea nach Druckatrophie zustande kommt, dann durch GJottiskrampf infolge doppelseitiger Rekurrensreizung.

\section{Die Struma intrathoracica.}

Uber intrathorakische Strumen und ihren Symptomenkomplex liegt eine große Literatur vor, jedoch sind die Abhandlungen meist sehr allgemein gehalten. Man hat weder die Strumenarten noch ihren Symptomenkomplex genau abgegrenzt, mithin hat man die letzte Schlußfolgerung nicht gezogen. In folgendem unterscheiden wir als Typen nach dem theoretischen Teil eine Struma intrapleuralis von einer Struma mediastinalis und hierbei wiederum eine Struma mëdiastinalis anterior und posterior.

Das Wort „intrapleuralis“ ist als Analogiebildung zu intraperitonealis aufzufassen und besagt nur, daß die Struma in mehr oder minder großer Ausdehnung von Pleura umgeben ist.

a) Die Struma intrapleuralis.

Fall 3. Mayer, Zur Lehreder Struma intrathora. cic a. Zentralbl. f. Chir. I919, Nr. 24. 
Aus dem Sektionsbericht: „Schilddruse auf beiden Seiten klein, im linken Lappen ein, im rechten Lappen zwei $1 \frac{1}{2} \mathrm{~cm}$ messende Knoten..." Rechte Lunge ziemlich stark verwachsen, Pleura über dem Oberlappen ziemlich stark verdickt, Oberlappen ziemlich voluminös, die Konsistenz in der oberen Hälfte stark vermehrt, in der unteren Hälfte und dem unscharf abgegrenzten Mittellappen normal. Auf Schnitt liegt im Oberlappen ein bis an die Pleura reichender 9:7:7 cm messender, ringsum gut abgekapselter Knoten. Der Rest des Oberlappens und der Mittellappen zeigen keine Veränderung außer Emphysem. Leichte Skoliose der Brustwirbelsäule. Bei genauer Untersuchung der in Formol fixierten rechten Lunge und der Halsorgane scheint bei äußerer Betrachtung die Pleura ununterbrochen über den Tumor hinwegzuziehen. Das Lungengewebe schiebt sich als schmaler Fortsatz zwischen die Pleura und die untere Hälfte des rundlichen Tumors. Die Lunge bewahrt so äußerlich durchaus ihre normale Form und füllte auch lückenlos den Thoraxraum aus. Auf der Schnittfläche erscheint der Tumor vom Lungengewebe durch eine dicke bindegewebige Kapsel, die in den seitlichen Partien kontinuierlich in die verdichtete Pleura übergeht, scharf abgegrenzt. Das Lungengewebe in unmittelbarer Nähe der Kapsel zeigt keinerlei Veränderungen.

Am oberen Pol des Tumors, zugleich der Lungenspitze, liegt außerhalb der Pleura bzw. der bindegewebigen Tumorkapsel, und mit dieser verwachsen eine sehr dünne Schicht von lappig gebautem, hellrotem Gewebe, das als dünner Gewebsstreifen hinter der A. und $V$. anonyma bis zum unteren Pol des rechten Schilddrüsenlappens zieht.“

Bei der Patientin, die an starker Kachexie und lobulärer Pneumonic starb, war vor 7 Jahren ein gutartiger Lungentumor diagnostiziert worden, über dessen Natur auch das Röntgenbild keinen Aufschluß gegeben hatte. Symptome von seiten des Tumors bestanden nicht.

In vorliegendem Falle war ein Knoten vom rechten Schilddrüsenlappen aus hinter $\operatorname{der} \mathrm{A}$. und $\mathrm{V}$. anonyma zur Lungenspitze gewachsen, hatte die Pleura eingestülpt und war von ihr allmählich wie in einem Beutel aufgenommen. Der rechte Oberlappen der Lunge war verdrängt und infolge der Volumabnahme der Lunge war eine vikariierende Erweiterung und später ein Emphysem der gesunden Partien entstanden. Hieraus erklärt sich die Skoliose der Brustwirbelsäule als Kompensationsskoliose. Subjektive Symptome konnten nicht vorhanden sein, da einmal ein sehr großer Teil der Lunge ohne Atemstörung infolge vikariierender Arbeit der gesunden Teile ausgeschaltet sein kann, dann 
aber innerhalb der Pleura außer der Lunge überhaupt keine Gebilde vorhanden sind, die lädiert werden können.

Der Fall beweist also, daB eine Struma intrapleuralisvon beträchtlicher Größe $(9: 7: 7 \mathrm{~cm})$ weder spezifische noch Allgemeinsymptome macht, falls es sich eben um eine reine Struma intrapleuralis handelt.

b) Die Struma mediastinalis.

Die Strumae mediastinales zerfallen in Strumae mediastinales anteriores und posteriores, von denen erstere, wie ausgeführt, nur bis zum Herzen wachsen, dem sie sich allmählich haubenförmig auflegen, letztere dagegen ungehindert das $Z$ werchfell erreichen können.

a) Die Struma mediastinalis anterior.

Fall 4. de Quervain, Spezielle chirurgische Diagnostik.

Eine 68jährige Frau, seit Jahren an Bronchitis leidend, kommt wegen hochgradiger Atemnot ins Spital. Sie kann nur in einer einzigen Stellung, sitzend vornüber gebeugt, einigermaßen atmen. Es besteht Dämpfung zu beiden Seiten des Sternums und das Röntgenbild ergibı einen dem Herzen haubenartig aufsitzenden, bis ins Jugulum reichenden, scharf abgegrenzten Schatten, dessen Form an ein Aneurysma denken läßt. Sein Rand pulsiert aber nicht und andere Anhaltspunkte fur diese Diagnose fehlen. Der rechte Schilddrüsenlappen enthält einige Kolloidknötchen. Vom linken Schilddrüsenlappen ist nichts zu fühlen als eine undeutliche Resistenz gegen das Jugulum hin. Es muß sich also um einen intrathorakischen Kropf handeln, auf dessen Rechnung auch die Bronchitis zu setzen ist. Die Operation bestätigte die Diagnose, und mit der Entfernung des Kropfes schwanden die Beschwerden sofort und bleibend.

In diesem Falle standen die Atemnot und die Bronchitis im Vordergrund, beides war durch Kompression der Trachea bedingt. Die Tatsache, daß Pat. nur in vornübergebeugter Stellung atmen konnte, beweist, daß die Kompression der Trachea von vorn ausgelöst wurde, indem der Tumor beim Vornüberbeugen sich dem Sternum näherte, und dadurch die Trachea freier wurde. Es handelte sich demnach um eine Struma mediastinalis anterior. Der Fall zeigt gleichzeitig, daß trotz der beträchtlichen Größe der Struma das Herz nicht disloziert worden ist. 
ß) Die Struma mediastinalis posterior.

Fall 5. Wuhrmann, Die Struma intrathoracica. Deutsche Zeitschr. f. Chir., Bd. 43. Mitgeteilt von Krönlein.

Aus der Anamnese: P. war seit seiner Kindheit engbrüstig. In späteren Jahren nahm die Engbrüstigkeit immer mehr zu und ebenso ein häufig sich einstellender Husten. Bergsteigen, später schon Treppensteigen verursachten große Beschwerden. Vor 30 Jahren beim Tanzen Erstickungsanfall, der sich dann alle Jahre einmal, später häufiger wiederholte. Zunahme der Atembeschwerden und des Hustenreizes in diesem Sommer. Ferner behauptet P. mit größter Bestimmtheit: Beim Husten bleibe ihm der Kehlkopf im Halse stecken, gehe nicht mehr herunter und er müsse ihn dann gewaltsam mit seinen Händen herunterdrücken, um nicht zu ersticken. Auch das Essen war in letzter Zeit erschwert.

Aus dem Status: Sämtliche Zeichen einer hochgradigen Tracheostenose. Für einen vermuteten, die Trachea komprimierenden Mediastinaltumor keine positiven Anhaltspunkte. Tracheotomie, wobei Tiefstand des Kehlkopfes auffällt. Vorübergehende Besserung, Patient stirbt bald unter allen Erscheinungen chronischer Kohlensäureintoxikation.

Aus dem Sektionsbericht: Von dem unteren und hinteren Pole des linken Lappens der im übrigen nicht vergrößerten Schilddruse geht ein herzförmig gestalteter, fast faustgroßer, glattwandiger, von einzelnen großen Venenästen begleiteter Fortsatz unter der ersten lin. ken Rippe nach unten in den Thorax, wo er sich medianwärts wendend zwischen Trachea und Ösophagus hineindrängt, mit seinem unteren Ende gerade bis zur Bifurkation der ersteren reichend und nach rechts den lateralen Rand von Ösophagus und Trachea wohl um $2 \mathrm{~cm}$ überragend. Die Länge dieses intrathorakischen Schilddrüsentumors beträgt $8 \mathrm{~cm}$, die Breite $7 \mathrm{~cm}$, die Dicke $5 \mathrm{~cm}$ und der Umfang $15 \mathrm{~cm}$. Der Tumor ist solide und stellt einen Kolloidkropf mit derber Kapsel dar. In der Höhe der oberen Thoraxapertur, entsprechend dem Druck der darüber liegenden linken ersten Rippe, trennt eine halsförmige Einschnürung den Tumor von der Hauptmasse des linken Schilddrüsenlappens. Durch die Einkeilung des Tumors zwischen Luft- und Speiseröhre sind diese beiden Organe in einer Längsausdehnung von $4-5 \mathrm{~cm}$, und zwar in der Höhe des Io. bis I2. Trachealringes $5 \mathrm{~cm}$ weit auseinandergedrängt, so zwar, daß die säbelscheidenartig abgeplattete und deutlich erweichte Trachea einen starken Bogen nach vorn und rechts beschreibt, während der Ösophagus ebenfalls platt gedrückt nach hinten und etwas nach links verdrängt erscheint. Erst an der Bifurkationsstelle der Trachea legen sich die beiden Organe in normaler Weise wieder aneinander. Genau entsprechend in Lage und Ausdehnung der intrathorakischen Struma zeigt die Wirbelsäule vom I. bis 5. Brustwirbel eine starke skoliotische Ver- 
krummung nach links. In der Konkavität dieser Krümmung liegt der Tumor.

Dieser Fall wird vollkommen beherrscht von den durch Kompression der Trachea ausgelösten Symptomen, nämlich von der Dyspnoe und den Erstickungsanfällen, die durch Verengerung des Trachealumens zustande kamen und dem Husten, der infolge des Druckes des Tumors auf die Trachea als Reizhusten aufzufassen ist. Diese beiden Symptome genügen, einen Mediastinaltumor auch bei Fehlen anderer objektiver Symptome als sicher annehmen zu lassen. Hätte man bei dem Falle, der vor der Röntgenära zur Beobachtung kam, ein Röntgenbild von vorn und von der Seite gemacht, so hätte man erstens den Tumor entdeckt und zweitens wahrscheinlich auch seine retrotracheale Lage infolge der Dislokation der Trachea diagnostiziert.

Die Tatsache, daß Pat. beim Husten der Kehlkopf im Halse stecken blieb und nicht mehr herunterging und er ihn dann gewaltsam herunterdrücken mußte, um nicht zu ersticken, erklärt sich aus der Einklemmung des Tumors mitsamt der Trachea in der oberen Thoraxapertur infolge der Hustenstöße.

Die Skoliose der Wirbelsäule rührt sicherlich von dem Druck des Tumors her, der sich schon in früher Kindheit entwickelt hatte.

\section{Zusammenfassung der Ergebnisse.}

\section{Die das Wachstum des Kropfes beeinflußenden Kräfte.}

Die das Wachstum des Kropfes beeinflussenden Kräfte sind

I. der Widerstand der Umgebung, der ihn in Richtung des geringsten Widerstands wachsen läßt, wie er sich aus den anatomischen Verhältnissen ergibt,

2. die eigene Schwere,

3. die Ansaugung durch den negativen Druck im Thorax bei geringem Wachstum nach unten,

4. artifizielle Momente, wie Halsbinde, unsachgemäße Behandlung usw.

\section{Die Ausbreitungsmöglichkeiten des Kropfes.}

1. Im allgemeinen.

Der Kropf kann bei Wachstum nach oben ungehindert durch das Spatium parapharyngeum in die Fossa retromandibularis ge- 
langen, bei Wachstum nach unten bieten ihm nach überwundener oberer Thoraxapertur erst die Organe des Mediastinums einen Widerstand. Bei Ausbreitung in horizontal-radiärer Richtung weisen die Muskellücken der Struma den Weg, die die Lücken verschließenden Gewebe, die Fascien, setzen der Ausbreitungsmöglichkeit eine Schranke.

2. Die speziellen Ausbreitungsmöglichkeiten des Kropfes.

a) In horizontal-radiärer Richtung.

Hier kommen als Durchtrittsräume nur das vordere Halsdreieck und der Spalt zwischen Sternocleidomastoideus und Scalenus anterior in Betracht, während die Scalenuslücken wegen ihrer geringen Größe fortfallen.

a) Wächst ein Kropf medial von den Scaleni nach hinten seitlich, so schmiegt er sich allmählich den Scaleni an und liegt hier fest wie in einer Nische, deren Boden von der oberen Thoraxapertur und den hier austretenden Gebilden dargestellt wird. Bei weiterem Wachstum schiebt er sich immer mehr medial und disloziert die locker fixierten Halsorgane.

ß) Wächst ein Kropf seitlich und etwas nach vorn, so schiebt er das Gefäßbündel des Halses beiseite und gleitet unter dem Kopfnicker in das seitliche Halsdreieck, wo er sich ungehindert entfalten kann.

r) Wächst ein Kropf median nach vorn, so überdehnt er die schwachen Gebilde des vorderen Halsdreiecks und hat uneingeschränkte Ausdehnungsmöglichkeit (Hängekropf).

b) Wachstum nach oben.

Es bietet keine Besonderheiten, da nicht das Wachstum nach oben, sondern die gleichzeitige Ausbreitung in horizontal-radiärer Richtung aussschlaggebend ist.

c) Wachstum nach unten.

a) Bei Wachstum nach unten seitlich tritt der Kropf meist hinter der A. und V. anonyma in Beziehung zur Lungenspitze. Er stülpt bei weiterem Wachstum die Pleura ein und wird von ihr allmählich wie in einem Beutel aufgenommen. 
$\beta$ ) Der in der Medianebene nach unten in das hintere Mediastinum wachsende Kropf kann ohne sonderlichen Widerstand das Zwerchfell erreichen.

r) Der ins vordere Mediastinum wachsende Kropf findet an dem Herzen, dem er sich allmählich haubenartig auflegt, eine unüberwindliche Schranke. Der Grund hierfür liegt einmal in der Einspannung der Struma in den Zug der Lungen, dann in der hämmernden Gegenbewegung des Herzens, die auch die tiefer liegenden Schichten verhindert, in Richtung des Herzens zu wachsen.

\section{Bezeichnung der Kropflagen.}

Man bezeichnet am besten:

I. den ins Spatium parapharyngeum und in die Fossa retromandibularis wachsenden Kropf als Struma parapharyngea und retromandibularis, den ins Spatium retropharyngeum wachsenden als Struma retropharyngea,

2. den medial von den Scaleni nach hinten seitlich wachsenden Kropf wegen seiner Fixation an den Scaleni als Struma colli lateralis posterior sive fixata,

3. den seitlich und nach vorn zwischen Sternocleidomastoideus und Scalenus anterior ins seitliche Halsdreieck wachsenden Kropf wegen seiner ungehinderten Ausbreitungsmöglichkeit als Struma colli lateralis anterior sive libera,

4. den zwischen den Sternocleidomastoidei nach vorn wachsenden Kropf als Struma colli anterior,

5. den nach seitlich unten zur Lungenspitze wachsenden Kropf, der allmählich von der Pleura wie in einem Beutel aufgenommen wird, als Struma intrapleuralis,

6. den ins vordere oder hintere Mediastinum wachsenden Kropf als Struma mediastinalis anterior und posterior.

\section{Literaturverzeichnis.}

I. Corning. Lehrbuch der topographischen Anatomie.

2. E is e ls b e rg, v., Die Krankheiten der Schilddrüse.

3. Klose, Die pathologisch-anatomischen Grundlagen der Basedowschen Krankheil.

4. Landois-Rosemann. Lehrbuch der Physiologie.

5. Mehring, v. Lehrbuch der inneren Medizin. 
I86 KLose, Die chirurgische Topographie des wachsenden Kropfes.

6. Mi y e $r$, Zur Lehre der Struma intrathoracica. Zentralbl. f. Chir. I9I9, Nr. 24.

7. Nordmann. Praktikum der Chirurgie.

8. Quervain, de. Spezielle chirurgische Diagnostik.

9. Rauber-Kopsch. Lehrbuch der Anatomie.

ıо. Sarbo, v., Zur Symptomatologie der intrathorakischen Struma. WViener klin. Wochenschr., Bd. 26, II.

I . Wölfler, Über dıe Entwicklung und den Bau des Kropfes.

I2. Wuhrmann, Die Struma intrathoracica. Deutsche Zeitschr. f. Chir., Bd. 43 . 